Supporting Information

\title{
Solvent choice in MOF linker exchange permits microstructural control
}

\author{
Ryan A. Dodson, ${ }^{\dagger}$ Andre P. Kalenak, ${ }^{\dagger}$ and Adam J. Matzger ${ }^{\star},+$, \\ ${ }^{\dagger}$ Department of Chemistry and $¥$ Macromolecular Science and Engineering Program, University \\ of Michigan, 930 North University Avenue, Ann Arbor, Michigan 48109-1055, United States
}

Table of contents:

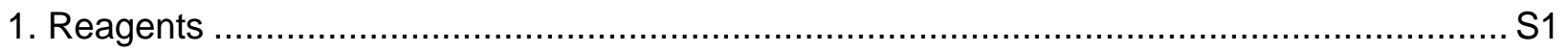

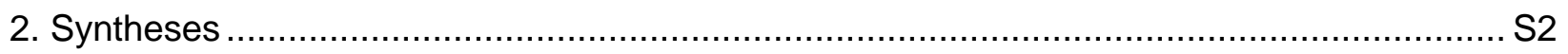

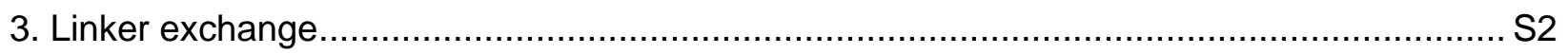

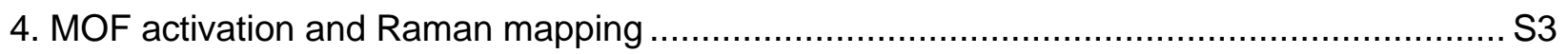

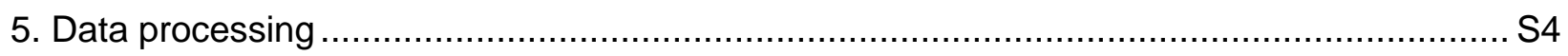

6. Linker solution concentration calculations ........................................................... 55

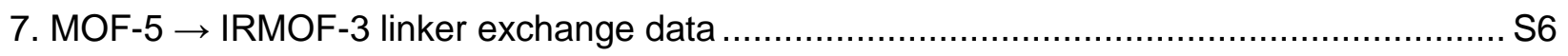

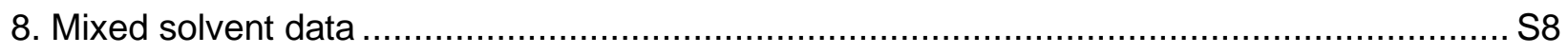

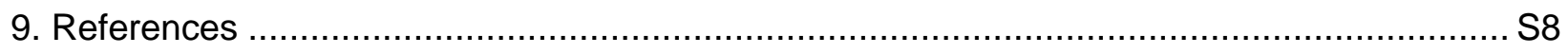

\section{Reagents}

Terephthalic acid $\left(\mathrm{H}_{2} \mathrm{bdc}\right.$, Fisher Scientific, 98\%), terephthalic acid- $d_{4}\left(\mathrm{H}_{2} \mathrm{bdc}-d_{4}\right.$, Sigma-Aldrich, 98\%, 98 atom \% D), and 2-aminoterephthalic acid $\left(\mathrm{H}_{2} \mathrm{bdc}-\mathrm{NH}_{2}\right.$, Sigma-Aldrich, 99\%) were used as received. Zinc nitrate hexahydrate (Fisher Scientific, ACS grade) was partially dehydrated by room temperature evacuation $(\sim 16 \mathrm{hr},<0.01$ Torr) to yield zinc nitrate tetrahydrate. $N, N$ Diethylformamide (DEF, Acros Organics, 99\%) was purified by storage on activated charcoal for $\sim 1$ month and passed through a silica gel column to remove impurities, followed by storage on activated $4 \AA$ molecular sieves. Acetonitrile (Fisher, Certified ACS $\geq 99.9 \%$ ), acetophenone (Acros Organics, 98\%), anisole (Acros Organics, 99.0\%), benzaldehyde (Sigma-Aldrich, 99.5\%), butanone (Fisher, ACS 99+\%), butyl acetate (Acros Organics, 99.5\%), cyclohexanone (Acros Organics, 99.8\%), dibutyl ether (Acros Organics, 99\%), 1,2-dimethoxyethane (Sigma-Aldrich, 99.5\%, inhibitor free), dimethyl carbonate (Acros Organics, 99\%), N,N-dibutylformamide (TCl Chemicals, >98.0\%), N,N-diethyl-3-methylbenzamide (Acros Organics, 98\%), N,Ndimethylacetamide (Acros Organics, 99\%), N,N-dimethylformamide (DMF, Fisher Scientific, 99.8\%), 2,5-dimethyltetrahydrofuran (Sigma-Aldrich, 98\%), dimethyl sulfoxide (DMSO, Fisher Scientific, HPLC grade), dioxane (Fisher, Certified ACS $\geq 99.9 \%$ ), ethyl acetate (Fisher, Certified ACS $\geq 99.5 \%$ ), 1-formylpiperdine (Acros Organics, 99\%), 1-formylpyrrolidine (Sigma-Aldrich, 97\%), $\mathrm{N}$-formylmorpholine (Acros Organics, $99+\%)$, methylene chloride $\left(\mathrm{CH}_{2} \mathrm{Cl}_{2}\right.$, Fisher scientific, 
ACS 99.5\%), $\mathrm{N}$-methylformanilide (Acros Organics, 99\%), $\mathrm{N}$-methyl-2-pyrrolidone (SigmaAldrich, 99.5\%), methyl $t$-butyl ether (Oakwood Chemical, 99\%), 2-methoxyethanol (Acros Organics, 99\%), $t$-butyl acetate (Sigma-Aldrich, $\geq 99 \%$ ), tetrahydrofuran (THF, Fisher Scientific, HPLC grade), propylene carbonate (Acros Organics, 99.5\%), and sulfolane (Oakwood Chemical, 99\%) were stored over activated $4 \AA$ molecular sieves after shaking for a minimum of 24 hours to minimize water content prior to use.

\section{Syntheses}

All MOF-5 syntheses were synthesized according to a modified published procedure. ${ }^{1}$ Specific synthetic protocols were as follows:

\section{MOF-5}

$\mathrm{H}_{2} \mathrm{bdc}$ (100.0 mg, $0.6019 \mathrm{mmol}, 1.0$ eq.) was combined with $\mathrm{Zn}\left(\mathrm{NO}_{3}\right)_{2} \cdot 4 \mathrm{H}_{2} \mathrm{O}(500.0 \mathrm{mg}, 1.912$ mmol, 3.2 eq.) along with $15 \mathrm{~mL}$ DEF in a $20 \mathrm{~mL}$ scintillation vial and dissolved. Synthesis was performed in a $100^{\circ} \mathrm{C}$ oven for 24 hours. Colorless cubic crystals $0.7-1.0 \mathrm{~mm}$ in size were obtained and washed $3 \times 15 \mathrm{~mL}$ with DMF.

$\mathrm{MOF}-5-d_{4}$

$\mathrm{H}_{2} \mathrm{bdc}-\mathrm{d}_{4}\left(20.5 \mathrm{mg}, 0.120 \mathrm{mmol}, 1.0\right.$ eq.) was combined with $\mathrm{Zn}\left(\mathrm{NO}_{3}\right)_{2} \cdot 4 \mathrm{H}_{2} \mathrm{O}(100.0 \mathrm{mg}, 0.3824$ mmol, 3.2 eq.) along with $3 \mathrm{~mL} D E F$ in a $4 \mathrm{~mL}$ scintillation vial and dissolved. Synthesis was performed in a $100^{\circ} \mathrm{C}$ oven for 24 hours. Colorless cubic crystals $0.7-1.0 \mathrm{~mm}$ in size were obtained and were washed $3 \times 3 \mathrm{~mL}$ with DMF.

$\mathrm{Zn}_{4} \mathrm{O}(\mathrm{bdc})_{1.5}\left(\mathrm{bdc}-\mathrm{d}_{4}\right)_{1.5}$

$\mathrm{H}_{2} \mathrm{bdc}$ (10.0 mg, $0.0602 \mathrm{mmol}, 0.5$ eq.), $\mathrm{H}_{2}$ bdc- $\mathrm{d}_{4}$ (10.2 mg, $0.0602 \mathrm{mmol}, 0.5$ eq.), and $\mathrm{Zn}\left(\mathrm{NO}_{3}\right)_{2} \cdot 4 \mathrm{H}_{2} \mathrm{O}(100.0 \mathrm{mg}, 0.3824 \mathrm{mmol}, 3.2$ eq.) were dissolved in $3 \mathrm{~mL} D E F$ in a $4 \mathrm{~mL}$ vial. Synthesis was performed in a $100{ }^{\circ} \mathrm{C}$ oven for 24 hours after which colorless cubic crystals 0.7 $1 \mathrm{~mm}$ in diameter were obtained and were washed $3 \times 3 \mathrm{~mL}$ with DMF.

\section{IRMOF-32}

$\mathrm{H}_{2}$ bdc- $\mathrm{NH}_{2}$ (100.0 mg, $0.5520 \mathrm{mmol}, 1.0$ eq.) was combined with $\mathrm{Zn}\left(\mathrm{NO}_{3}\right)_{2} \cdot 6 \mathrm{H}_{2} \mathrm{O}(474.0 \mathrm{mg}$, $1.593 \mathrm{mmol}$, 2.9 eq.) along with $10 \mathrm{~mL}$ DMF in a $20 \mathrm{~mL}$ scintillation vial and dissolved, and then split into $2 \mathrm{~mL}$ aliquots in 10 separate $4 \mathrm{~mL}$ vials. Synthesis was performed in a $100^{\circ} \mathrm{C}$ oven for 16 hours. Yellow cubic crystals were obtained and washed $3 \times 3 \mathrm{~mL}$ with DMF.

\section{Linker exchange}

$10 \mathrm{mM}$ solutions of $\mathrm{H}_{2} \mathrm{bdc}-d_{4}$ were prepared by sonicating $17.0 \mathrm{mg} \mathrm{H}_{2} \mathrm{bdc}-d_{4}$ with $10 \mathrm{~mL}$ of solvent in a $20 \mathrm{~mL}$ scintillation vial until full dissolution was achieved. In cases where full dissolution did not result, the solutions were instead sonicated until no further dissolution was observed ( 30-60 min.), then used without filtration of excess solid $\mathrm{H}_{2} b d c-d_{4}$ in order to avoid varying the molar ratio of bdc:bdc- $d_{4}$ between experiments. 
After preparation of the $\mathrm{H}_{2}$ bdc- $d_{4}$ solutions, $\sim 18 \mathrm{mg}$ aliquots (see Section 6 ) of MOF-5 stored in DMF were collected and transferred into fresh vials, excess DMF was removed via pipette, and the $\mathrm{H}_{2}$ bdc- $d_{4}$ solutions were added to each. Vials were shaken on a VXR Basic Vibrax orbital shaker (IKA) at 500 RPM, keeping MOF crystals suspended in solution while minimizing mechanical abrasion. After 24 hours, aliquots of the exchanged MOF were removed and then washed $3 \times 3 \mathrm{~mL}$ (15 minutes between washes) with DMF to remove excess linker remaining in the MOF pores.

13 solvents were chosen of the original 30 for further characterization. These included the amides (DMF, N,N-diethylformamide, $N, N$-dibutylformamide, $N$-methyl-2-pyrrolidone, 1-formylpiperdine, 1 -formylpyrrolidine, $\mathrm{N}$-formylmorpholine, $\mathrm{N}$-methylformanilide, $\mathrm{N}, \mathrm{N}$-diethyl-3-methylbenzamide), THF, dioxane, butyl acetate, and propylene carbonate. These solvents were chosen because they showed evidence of bdc- $d_{4}$ incorporation after exchange (based on Raman spectra of the crystal surfaces), and because they dissolved either all or a significant fraction of the $10 \mathrm{mM} \mathrm{H}_{2} \mathrm{bdc}-d_{4}$. There is potential for further characterization of the other solvents that showed exchange without degrading MOF-5 or fully dissolving $10 \mathrm{mM} \mathrm{H}_{2}$ bdc- $d_{4}$ (ethyl acetate, $t$-butyl acetate, butanone, acetophenone, 2,5-dimethyltetrahydrofuran, dibutyl ether, dimethyl carbonate), but only dioxane, propylene carbonate, and butyl acetate were chosen from this category in this work.

For PSE with mixed solvents (DMF/N,N-diethyl-3-methylbenzamide and $N, N$ diethylformamide/ $\mathrm{N}, \mathrm{N}$-diethyl-3-methylbenzamide) the same general protocol was utilized, but with the addition of two solvents in the linker dissolution step rather than one, to a total volume of $10 \mathrm{~mL}$. Similarly, for the MOF-5 $\rightarrow$ IRMOF-3 PSE, the only adjustments to the above protocol were the use of $\mathrm{H}_{2}$ bdc $-\mathrm{NH}_{2}(18.1 \mathrm{mg})$ instead of $\mathrm{H}_{2} \mathrm{bdc}-d_{4}$, and the use of either $N, N$ diethylformamide or $\mathrm{N}$-methyl-2-pyrrolidone as solvents.

\section{MOF activation and Raman mapping}

From DMF, crystals were washed $3 \times 3 \mathrm{~mL}$ with methylene chloride $\left(\mathrm{CH}_{2} \mathrm{Cl}_{2}\right)$ at 15-minute intervals. Following the final $\mathrm{CH}_{2} \mathrm{Cl}_{2}$ exchange, excess solvent was decanted, and the material was activated under dynamic vacuum (<10 mTorr) for 16 hours at room temperature. Activated material was transferred to a desiccator for storage.

Activated MOF was transferred from the desiccator to a $20 \times 20 \times 0.5 \mathrm{~mm}$ polished steel substrate. Upon transfer, the material was sorted by crystal quality and cleaved by hand parallel to the (100) faces of the crystals with a steel razor blade. Aligning the cleavage plane parallel to a crystal face was imperative to achieving clean edges in Raman mapping analysis. Crystals with visible defects were avoided for their tendency to crumble under the razor. The newly cleaved face of the crystal was then oriented upwards and the edges, as accurately as possible, were aligned with the $X$ and $Y$ axes of the instrument mapping stage. Raman mapping microspectroscopy was carried out on a Renishaw InVia Qontor confocal Raman microscope equipped with a Renishaw Centrus 2957 detector, a Leica $50 \times$ long working distance objective, and a $785 \mathrm{~nm}$ laser. The stage enclosure was supplied with positive pressure of dry air to mitigate MOF degradation during collection, resulting in a relative humidity consistently $<1 \%$. Preliminary spectra were acquired with a $510-$ $1650 \mathrm{~cm}^{-1}$ window (Raman shift) at 10 accumulations of $0.5 \mathrm{~s}$ exposures, typically one near the crystal edge and another $\sim 100 \mu \mathrm{m}$ from the edge to confirm the cleaved face was exposed. Maps were collected with similar spectral parameters $\left(510-1650 \mathrm{~cm}^{-1}\right.$ window, $0.5 \mathrm{~s}$ exposure, single accumulation) in an array spanning $\sim 100 \times 15 \mu \mathrm{m}$ with 0.1 and $2 \mu \mathrm{m}$ resolution in the orthogonal and parallel axis (respective to the edge). 


\section{Data processing}

Raw spectral data were converted to 1D extent of exchange versus depth profiles using WiRE 5.3 (Renishaw) followed by data normalization with custom-written Python code. First, spectra saturated by cosmic rays were removed, subjected to a zero-order background subtraction, then deconvoluted into MOF-5 and MOF-5- $d_{4}$ signals using Non-Negative Least Squares (NNLS) component analysis as implemented in WiRE 5.3. Briefly, for a two-component system, NNLS minimizes the difference (via the method of least squares) between the spectrum and the sum of two scaled reference spectra, with the constraint that the scaling factors cannot be negative. The reference spectra used in NNLS were experimentally measured Raman spectra of MOF-5 and MOF-5- $d_{4}$. These spectra were previously scaled relative to one another such that the difference between their adjusted sum and the measured Raman spectrum of $\mathrm{Zn}_{4} \mathrm{O}(\mathrm{bdc})_{1.5}\left(\mathrm{bdc}-d_{4}\right)_{1.5}$ was minimized. Thus, $\mathrm{Zn}_{4} \mathrm{O}(\mathrm{bdc})_{1.5}\left(\mathrm{bdc}-d_{4}\right)_{1.5}$ acts as the basis for the calibration.

After NNLS, line maps were normalized based on several facts about the system. Because it is a mixed isostructural material, the products of the MOF-5 $\rightarrow$ MOF-5- $d_{4}$ linker exchange should spectroscopically appear as a linear combination of MOF-5 and MOF-5- $d_{4}$. Put another way, for any Raman scan $S_{i}$ of the system, $S_{i, \text { total }}=\left(a_{i} \times S_{i, b d c}\right)+\left(b_{i} \times S_{i, b d c-d 4}\right)$. The crystal should have effectively uniform density, so for every scan (assuming the same volume of sample is probed), $a_{i}+b_{i}=c$, where $c$ is a constant defined here as unity. Deviations from this prediction result from surface inhomogeneities, which the data must be normalized to correct for. This can be simply done by dividing each $a_{i}$ and $b_{i}$ by $\left(a_{i}+b_{i}\right)$; however, care must be taken near the crystal edges.

The exchanged linker bdc- $d_{4}$ should be most concentrated at the crystal surface, as this is where the least diffusion (and thus time) is required for exchange. This would ideally translate to the maximum bdc- $d_{4}$ Raman signal occurring exactly at the crystal edge. However, in practice the bdc- $d_{4}$ signal is highest at the closest position to the crystal edge where irradiation of the crystal surface is maximized (Figure S1). As the laser scans further from this point, is begins to transition off of the crystal, and less of the sample is irradiated and therefore detected. Thus, away from the crystal center from the point of maximum bdc- $d_{4}$ intensity (e.g. at points 1 and 2 in Figure S1 A), the assumption that $a_{i}+b_{i}=c$ is no longer true. To address this boundary condition, the assumption is made that the scaling factor $\left(a_{i}+b_{i}\right)$ for scans earlier than the maximum bdc- $d_{4}$ point is equal to the scaling factor at that point, $\left(a_{d 4-\max }+b_{d 4-\max }\right)$. This simplification is reasonable because the remaining surface can be assumed to be nearly homogeneous over such a small region.

A)

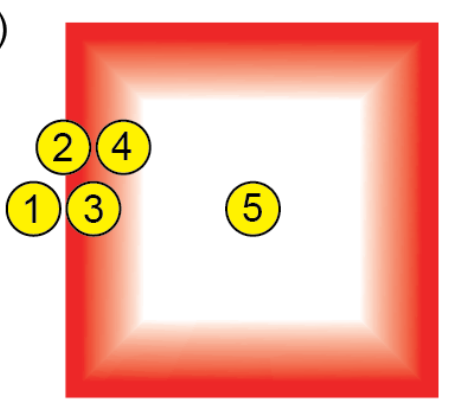

B)

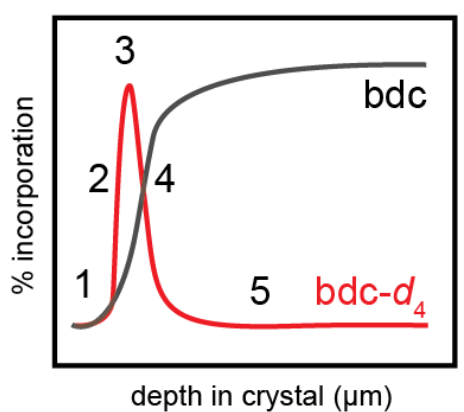

Figure S1. Relationship between laser position on sample and observed linker incorporation for a model core-shell system. A) Model MOF-5 $\rightarrow$ MOF-5- $d_{4}$ core-shell system (higher bdc- $d_{4}$ density shown in red) with five example laser spot locations indicated. B) Idealized percent incorporation versus depth in the crystal for the model in (A). Each of the five sample laser spot locations are shown on the plot. 


\section{Linker solution concentration calculations}

Because PSE involves exchanging linker molecules between the solution and the MOF, it is important to consider the maximum degree to which the solution composition can vary over the course of the exchange. Particularly, if PSE in a particular solvent depletes solution $\mathrm{H}_{2} \mathrm{bdc}-d_{4}$ (and thus equilibrates with the solution linker) more rapidly than PSE in other solvents, there is the potential for underestimating the exchange rate in the fast conditions, as the process will be slowed by the decreased exchanging linker concentration in solution. Upper, middle, and lower estimates for the percentage of bdc- $d_{4}$ incorporated in the MOF (and $\mathrm{H}_{2}$ bdc- $d_{4}$ lost from the solution) are as follows:

In these PSE studies, $10 \mathrm{~mL}$ quantities of $10 \mathrm{mM} \mathrm{H}_{2}$ bdc- $d_{4}$ solutions were used. An estimate for the quantity of MOF-5 used in each experiment was determined by transferring three aliquots of DMF-solvated MOF into three empty vials, and then washing and activating the material according to the protocol described above in Section 4. This yielded an average mass of MOF-5 of $18.1 \pm$ $0.9 \mathrm{mg}$. This can be converted into moles of bdc in MOF-5 added at the start of the exchange.

$18.1 \pm 0.9 \mathrm{mg} \mathrm{MOF}-5 \times \frac{1 \mathrm{mmol} \mathrm{Zn}_{4} \mathrm{O}(\mathrm{bdc})_{3}}{769.46 \mathrm{mg} \mathrm{MOF}-5} \times \frac{3 \mathrm{mmol} \mathrm{bdc}}{1 \mathrm{mmol} \mathrm{Zn}_{4} \mathrm{O}(\mathrm{bdc})_{3}}=0.0706 \pm 0.0035 \mathrm{mmol} \mathrm{bdc}$

To make each of the $10 \mathrm{mM} 10 \mathrm{~mL}$ solutions, $17.0 \mathrm{mg}$ of $\mathrm{H}_{2} \mathrm{bdc}-d_{4}$ was used, corresponding to $0.100 \mathrm{mmol}$ of $\mathrm{H}_{2} \mathrm{bdc}-d_{4}$. Thus, at the extreme case of $19.0 \mathrm{mg}$ of MOF-5 added, the starting $\mathrm{H}_{2}$ bdc- $d_{4}:$ bdc ratio in the system is

$$
\frac{0.100 \mathrm{mmol} \mathrm{H}_{2} \mathrm{bdc}-\mathrm{d}_{4}}{0.0741 \mathrm{mmol} \mathrm{bdc}}=1.35 \mathrm{H}_{2} \mathrm{bdc}-\mathrm{d}_{4} \text { per bdc }
$$

Of the solvents tested, $N, N$-diethylformamide gave the highest overall extent of exchange. The MOF- 5 crystals ranged from $700-1000 \mu \mathrm{m}$ in diameter, but the greatest perturbation to the linker solution composition would occur with the smallest sized crystals, so a worst-case scenario for $\mathrm{H}_{2}$ bdc- $d_{4}$ depletion can be calculated with the assumption that all crystals are $700 \mu \mathrm{m}$ in diameter. $N, N$-Diethylformamide gave $56 \mu \mathrm{m}$ shells on average, with maximum extents of exchange of $\sim 66$ $\%$ : for these calculations, it will be assumed that $60 \mu \mathrm{m}$ shells with uniform $70 \%$ exchange resulted. Such an exchange would result in the crystals being $30.2 \%$ bdc- $d_{4}$ overall, and $22.4 \%$ of all $\mathrm{H}_{2} \mathrm{bdc}-d_{4}$ in solution would be replaced by $\mathrm{H}_{2} \mathrm{bdc}$, decreasing $\left[\mathrm{H}_{2} \mathrm{bdc}-d_{4}\right]$ from $10 \mathrm{mM}$ to 7.76 $\mathrm{mM}$.

DMF is a more representative example of a high exchanging solvent. Assuming $18.1 \mathrm{mg}$ of 850 $\mu \mathrm{m}$ MOF-5 crystals, $20 \mu \mathrm{m}$ thick shells, and uniform $65 \%$ exchange, $8.8 \%$ of the linkers in the MOFs would be bdc- $d_{4}$, and $6.2 \%$ of $\mathrm{H}_{2}$ bdc- $d_{4}$ in solution would be replaced with $\mathrm{H}_{2} \mathrm{bdc}$, giving a final $\left[\mathrm{H}_{2} \mathrm{bdc}-\mathrm{d}_{4}\right]$ of $9.38 \mathrm{mM}$.

A low exchanging solvent such as 1 -formylpyrrolidine, with $17.2 \mathrm{mg}$ of $1000 \mu \mathrm{m}$ MOF-5 crystals, $6 \mu \mathrm{m}$ thick shells, and uniform $25 \%$ exchange, would give an exchanged product with $0.9 \%$ bdc$d_{4}$, and would use $0.6 \%$ of all $\mathrm{H}_{2}$ bdc- $d_{4}$ in the starting solution, resulting in a final $\left[\mathrm{H}_{2} \mathrm{bdc}-d_{4}\right]$ of $9.99 \mathrm{mM}$. 


\section{MOF-5 $\rightarrow$ IRMOF-3 linker exchange data}
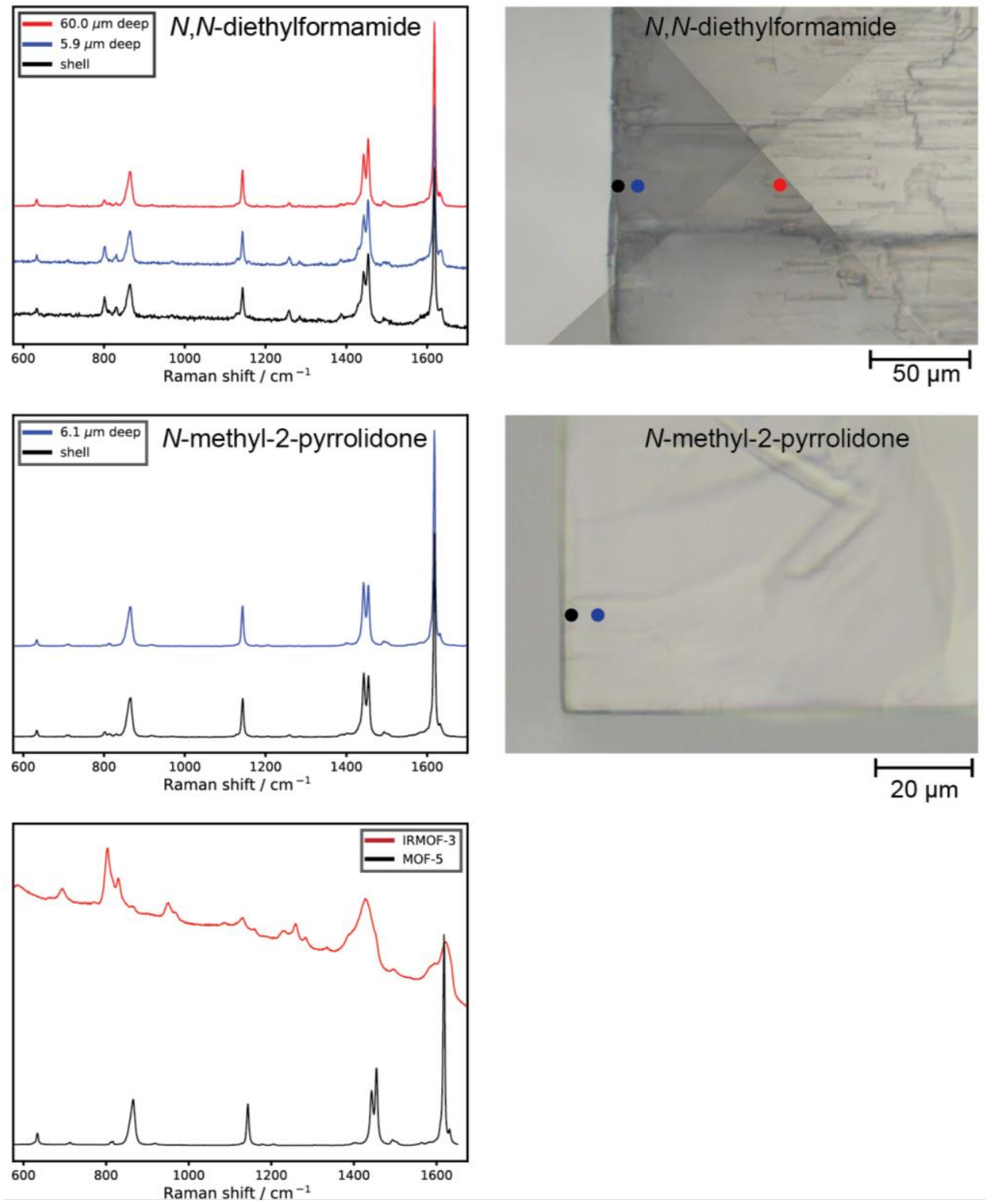

Figure S2. Comparison of $N, N$-diethylformamide and $N$-methyl-2-pyrrolidone in facilitating the MOF-5 $\rightarrow$ IRMOF-3 linker exchange. Left, above: Raman spectra of cross-sectioned MOF resulting from the MOF-5 $\rightarrow$ IRMOF-3 linker exchange in $N, N$-diethylformamide. Spectra taken at the far edge of the shell, $5.9 \mu \mathrm{m}$ deep in the crystal, and $60.0 \mu \mathrm{m}$ deep in the crystal are shown. Left, middle: Raman spectra of cross-sectioned MOF resulting from the MOF-5 $\rightarrow$ IRMOF-3 linker exchange in $N$-methyl-2-pyrrolidone. Spectra taken at the far edge of the shell and $6.1 \mu \mathrm{m}$ deep in the crystal are shown. Left, below: Raman spectra of pure phase MOF-5 and IRMOF-3. Right, above: locations of Raman spectra collected of the sample resulting from the MOF- $5 \rightarrow$ IRMOF3 linker exchange in $\mathrm{N}, \mathrm{N}$-diethylformamide. Visual artifacts in the image (discontinuities in image brightness) result from the automated image stitching process in WiRE 5.3 rather than from the sample itself. Right, below: locations of Raman spectra collected of the sample resulting from the MOF-5 $\rightarrow$ IRMOF-3 linker exchange in $N$-methyl-2-pyrrolidone. 

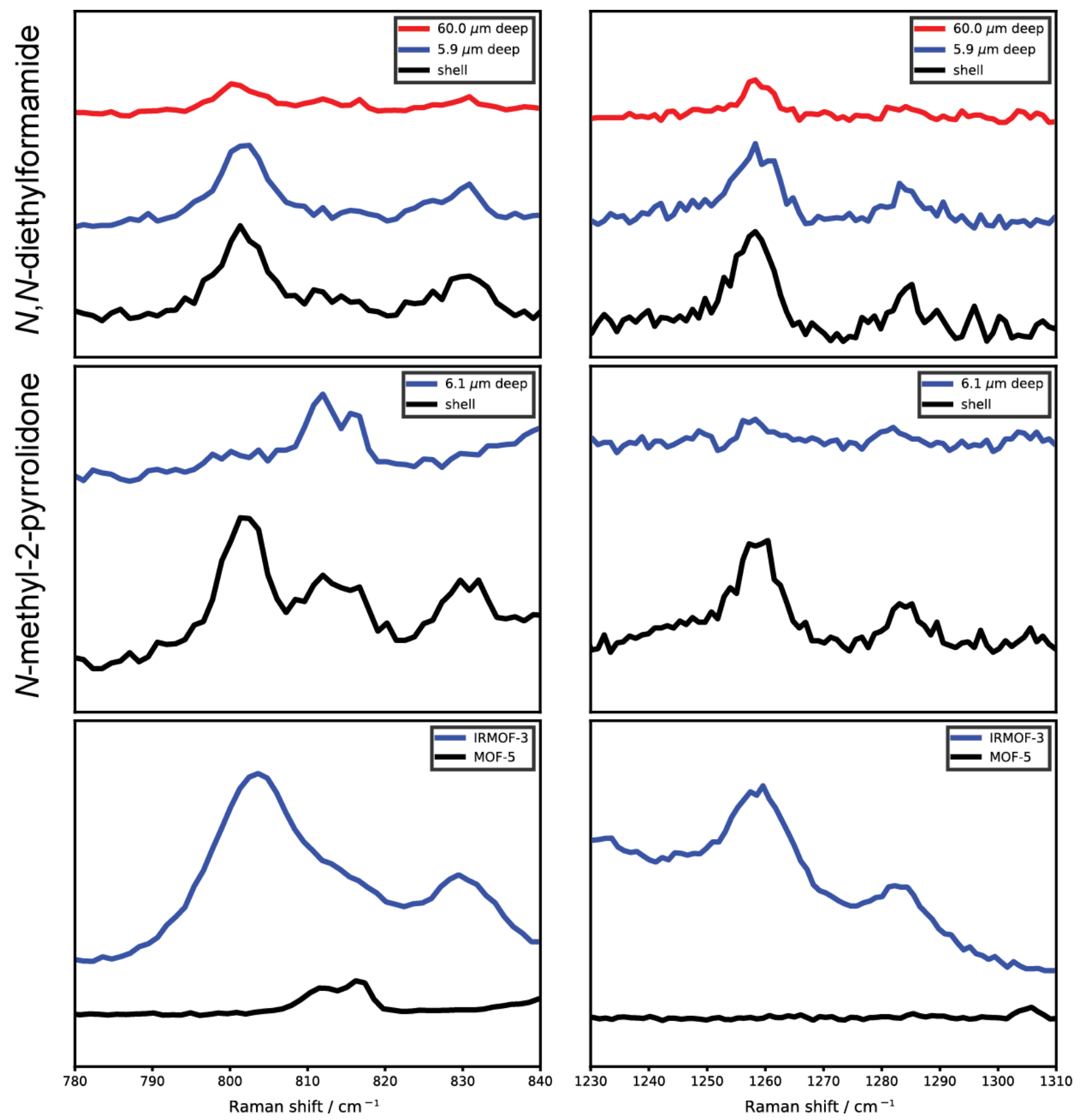

Figure S3. Magnified regions of interest from Raman spectra given in Figure S2 of the MOF-5 $\rightarrow$ IRMOF-3 linker exchange. Left: $780-840 \mathrm{~cm}^{-1}$ region. Right: $1230-1310 \mathrm{~cm}^{-1}$ region. Above: Raman spectra of cross-sectioned MOF resulting from the MOF-5 $\rightarrow$ IRMOF-3 linker exchange in $N, N$-diethylformamide. Spectra taken at the far edge of the shell, $5.9 \mu \mathrm{m}$ deep in the crystal, and $60.0 \mu \mathrm{m}$ deep in the crystal are shown. Exchange at least as deep as $60 \mu \mathrm{m}$ is observed. Middle: Raman spectra of cross-sectioned MOF resulting from the MOF- $5 \rightarrow$ IRMOF3 linker exchange in $\mathrm{N}$-methyl-2-pyrrolidone. Spectra taken at the far edge of the shell and 6.1 $\mu \mathrm{m}$ deep in the crystal are shown. Exchange is observed at the surface, but is minimal $6.1 \mu \mathrm{m}$ deep in the crystal. Below: Raman spectra of phase pure MOF-5 and IRMOF-3. 


\section{Mixed solvent data}

Table S1. Measured shell parameters for mixed solvents. Degree of shell exchange indicates the maximum degree of exchange measured in the shell. Thickness is given as shell full-width at half-max. Values are given as the average \pm the standard error of the mean $(\sigma / \sqrt{ } n)$.

\begin{tabular}{|l|c|c|}
\hline \multicolumn{1}{|c|}{ Solvent } & Degree of shell exchange $(\%)$ & Shell thickness $(\mu \mathrm{m})$ \\
\hline $\begin{array}{l}\text { 1:3 } N, N \text {-diethyl-3- } \\
\text { methylbenzamide:DMF }\end{array}$ & $65.8 \pm 1.2$ & $14.6 \pm 0.5$ \\
\hline $\begin{array}{l}1: 1 N, N \text {-diethyl-3- } \\
\text { methylbenzamide:DMF }\end{array}$ & $57.8 \pm 1.2$ & $9.3 \pm 0.6$ \\
\hline $\begin{array}{l}3: 1 N, N \text {-diethyl-3- } \\
\text { methylbenzamide:DMF }\end{array}$ & $41.5 \pm 1.9$ & $4.0 \pm 0.2$ \\
\hline $\begin{array}{l}\text { 9:1 } N, N \text {-diethyl-3- } \\
\text { methylbenzamide:DMF }\end{array}$ & $8.4 \pm 0.7$ & $3.1 \pm 0.2$ \\
\hline $\begin{array}{l}1: 3 N, N \text {-diethyl-3- } \\
\text { methylbenzamide: } N, N- \\
\text { diethylformamide }\end{array}$ & & $4.0 \pm 0.4$ \\
\hline $\begin{array}{l}1: 1 N, N \text {-diethyl-3- } \\
\text { methylbenzamide: } N, N- \\
\text { diethylformamide }\end{array}$ & $41.1 \pm 1.3$ & $6.0 \pm 0.6$ \\
\hline $\begin{array}{l}3: 1 N, N \text {-diethyl-3- } \\
\text { methylbenzamide: } N, N- \\
\text { diethylformamide }\end{array}$ & $16.3 \pm 2.9$ & $4.3 \pm 0.4$ \\
\hline
\end{tabular}

\section{References}

(1) Li, H.; Eddaoudi, M.; O’Keeffe, M.; Yaghi, O. M. Design and synthesis of an exceptionally stable and highly porous metal-organic framework. Nature 1999, 402, 276.

(2) Eddaoudi, M.; Kim, J.; Rosi, N.; Vodak, D.; Wachter, J.; O’Keeffe, M.; Yaghi, O. M. Systematic Design of Pore Size and Functionality in Isoreticular MOFs and Their Application in Methane Storage. Science 2002, 295, 469. 